\title{
Existence of Minimizers and Microstructure in Nonlinear Elasticity
}

\author{
Zhiping Li \\ Department of Mathematics, Peking University, \\ Beijing 100871, People's Republic of China
}

\begin{abstract}
In this paper, the existence of a solution in the form of a minimizer or microstructure is established for the boundary value problems of nonlinear elasticity with certain nonconvex stored energy functions such as those of St. Venant-Kirchhoff type materials. Necessary and sufficient conditions for minimizing sequences of the potential energy to converge to a minimizer or to microstructure are given.
\end{abstract}

Key words: existence, minimizer, microstructure, nonlinear elasticity, minimizing sequence, convergence

\section{Introduction}

In this paper, the existence of solutions in the form of minimizers or microstructure [1] [2] is established for the boundary value problems of nonlinear hyperelasticity with certain nonconvex stored energy functions. The study was motivated by the consideration of nonlinear elastic materials whose stored energy functions are of the form [3]

$$
W(F) \equiv W^{*}(E)=\frac{\lambda}{2}(\operatorname{tr} E)^{2}+\mu \operatorname{tr} E^{2}+o\left(|E|^{2}\right),
$$

where $F=I+\nabla u$ with $\nabla u$ being the displacement gradient and $I$ being the $3 \times 3$ identity matrix,

$$
E=\frac{1}{2}\left(F^{T} F-I\right)=\frac{1}{2}\left(\nabla u^{T}+\nabla u+\nabla u^{T} \nabla u\right)
$$


is the strain tensor and $\lambda>0, \mu>0$ are Lamé's constants.

As far as what is known to the author, for such materials, the existence theorem is only established, by using the implicit function theorem, for the case when the pure displacement boundary value condition is considered and the body force is sufficiently small (see [3] [4] [5]), and there is no way to extend this approach to the mixed boundary value problems.

Ball's theory on polyconvexity provides an important approach to the existence theorems for general boundary value problems of nonlinear elasticity [6] [7] [8]. But, the theory does not apply to the stored energy functions of the form (1.1), since they are in general neither polyconvex nor quasiconvex (see [9]). Recently, developements have been made on quasiconvex envelopes of stored energy functions which leads to the consideration of relaxed minimization problem instead of the original one (see [10] [11] [12] [13] [14] among others). But this approach rules out a natural reqirement in nonlinear elasticity that stored energy functions are such that

$$
W(F)=+\infty, \quad \text { if } \operatorname{det} F \leq 0,
$$

and

$$
W(F) \rightarrow+\infty, \quad \text { as } \operatorname{det} F \rightarrow 0^{+} .
$$

In addition, it may fail to show some important features of minimizing sequences of the original problem, especially when the infinimum can not be reached.

In this paper, a generalized polyconvexity conditions for stored energy functions are introduced (see (H1) - (H3) in §2), and the existence of solutions in the form of minimizers or microstructures, which are characterized by minimizing sequences, is established for the boundary value problems of nonlinear elasticity with stored energy functions satisfying these conditions (see $\S 2$ ). This generalizes Ball's existence theory for polyconvex materials [7] [8] since the generalized polyconvexity conditions $((\mathrm{H} 1)-(\mathrm{H} 2)$ in $\S 2)$ include the polyconvexity conditions as a special case. Stored energy functions satisfying the generalized polyconvexity conditions are in general neither polyconvex nor quasiconvex.

In $\S 2$, necessary and sufficient conditions for a minimizing sequence to converge to a minimizer or to microstructure are also obtained. These conditions are useful in computing the solutions.

Applying the theory established in $\S 2$ to nonlinear elastic materials, we see that there always exists a solution in the form of either a minimizer or microstructure for the boundary value problems if $o\left(|E|^{2}\right)$ in (1.1) 
satisfies generalized polyconvexity and certain growth conditions (see $\S 3)$. The theory can also be adjusted to cover (see §2) St. VenantKirchhoff materials whose stored energy functions are

$$
W_{1}(E)=\frac{\lambda}{2}(\operatorname{tr} E)^{2}+\mu \operatorname{tr} E^{2},
$$

which obviously do not satisfy hypothesis (H3) and hence (1.3) and (1.4). Hence we are able to claim that for boundary value problems of St. Venant-Kirchhoff materials, every minimizing sequence contains a subsequence which either converges strongly to a minimizer of the total potential energy or leads to microstructure (see Remark 2.4).

\section{Existence of solutions and convergence of minimizing sequences}

Let $\Omega \subset R^{3}$ be a connected open set with Lipschitz continuous boundary $\partial \Omega$. Let $\partial \Omega_{0} \subset \partial \Omega, \partial \Omega_{1} \subset \partial \Omega, \partial \Omega_{0} \cap \partial \Omega_{1}=\emptyset$ and area $\left(\partial \Omega_{0}\right) \neq 0$.

Let the stored energy function $W(x, F)$ satisfy the following hypotheses:

(H1) There is a Carathéodory function $G: \bar{\Omega} \times M^{3} \times M^{3} \times M^{3} \times$ $R_{+} \rightarrow$ R, i.e. $G(x, \cdot, \cdot, \cdot, \cdot)$ is continuous for almost all $x \in \bar{\Omega}$ and $G(\cdot, E, F, H, \delta)$ is measurable for all $(E, F, H, \delta) \in M^{3} \times M^{3} \times M^{3} \times$ $R_{+}$, such that $G(x, \cdot, \cdot, \cdot, \cdot)$ is strictly convex and

$$
W(x, F)=G(x, E, F, \operatorname{adj} F, \operatorname{det} F), \quad \text { for all } x \in \bar{\Omega} \text { and } F \in M_{+}^{3},
$$

where $M^{3}=\{$ all $3 \times 3$ matrices $\}, M_{+}^{3}=\left\{F \in M^{3}: \operatorname{det} F>0\right\}$, $E=\frac{1}{2}\left(F^{T} F-I\right)$ with $I$ being the $3 \times 3$ identity matrix, adj $F$ is the transpose of the matrix of cofactors of $F$ and $\operatorname{det} F$ is the determinant of $F$.

(H2) There are constants $C_{0} \in R, C_{1}>0, s>1, p \geq 2 s, q \geq \frac{p}{p-1}$, $r>1$ such that

$$
G(x, E, F, H, \delta) \geq C_{0}+C_{1}\left(|E|^{s}+|F|^{p}+|H|^{q}+|\delta|^{r}\right),
$$

for all $(x, E, F, H, \delta) \in \bar{\Omega} \times M^{3} \times M^{3} \times M^{3} \times R_{+}$. 
(H3)

$$
G\left(x, E_{n}, F_{n}, H_{n}, \delta_{n}\right) \longrightarrow+\infty
$$

if $\delta_{n} \rightarrow 0^{+}$.

To simplify the notation, we denote, for $x \in \Omega, E \in M^{3}$ and $F \in M^{3}$,

$$
Z(x, E, F)= \begin{cases}G(x, E, F, \operatorname{adj} F, \operatorname{det} F), & \text { if } \operatorname{det} F>0 ; \\ +\infty, & \text { if } \operatorname{det} F \leq 0 .\end{cases}
$$

Then, it is obvious that for $F \in M_{+}^{3}$

$$
W(x, F)=Z(x, E, F), \quad \text { where } \quad E=\frac{1}{2}\left(F^{T} F-I\right) .
$$

We consider the problem of minimizing the functional

$$
I(u)=\int_{\Omega} W(x, I+\nabla u) d x-\int_{\Omega} f \cdot u d x-\int_{\partial \Omega_{1}} g \cdot u d s,
$$

which is the total energy of the elastic body subject to a body force $f$, a traction $g$ and at displacement $u$, in the set of admissible functions

$$
\begin{aligned}
& A=\left\{u \in W^{1, p}\left(\Omega ; R^{3}\right): \operatorname{adj}(I+\nabla u) \in L^{q}\left(\Omega ; M^{3}\right),\right. \\
& \operatorname{det}(I+\nabla u) \in L^{r}(\Omega), \operatorname{det}(I+\nabla u)>0 \text {, a.e. in } \Omega \text {, } \\
& \text { and } \left.u=u_{0} \text { on } \partial \Omega_{0}\right\} \text {, }
\end{aligned}
$$

where $f$ and $g$ are in such function spaces that

$$
\Phi(u)=\int_{\Omega} f \cdot u d x-\int_{\partial \Omega_{1}} g \cdot u d s
$$

defines a continuous functional in $W^{1, p}\left(\Omega ; R^{3}\right)$ and area $\left(\partial \Omega_{0}\right)>0$.

Lemma 2.1 : Let $W(x, F)$ satisfy (H1) - (H3). Let $\Phi(u)$, defined by (2.4), be continuous in $W^{1, p}\left(\Omega ; R^{3}\right)$.

Then, there exist constants $\alpha_{0} \in R$ and $\alpha_{1}>0$ such that

$$
I(u) \geq \alpha_{0}+\alpha_{1}\left(\|E\|_{s}^{s}+\|F\|_{p}^{p}+\|\operatorname{adj} F\|_{q}^{q}+\|\operatorname{det} F\|_{r}^{r}\right), \quad \forall u \in A,
$$

where

$$
F=I+\nabla u
$$

and

$$
E=\frac{1}{2}\left(F^{T} F-I\right)
$$


Proof: By (H2), we have

$$
\int_{\Omega} W(x, F) d x \geq \alpha+\beta\left(\|E\|_{s}^{s}+\|F\|_{p}^{p}+\|a d j F\|_{q}^{q}+\|\operatorname{det} F\|_{r}^{r}\right)
$$

for some constants $\alpha \in R$ and $\beta>0$.

It follows from the continuity of $\Phi(\cdot)$ in $W^{1, p}\left(\Omega ; R^{3}\right)$, Poincaré inequality [15] and Hölder inequality that

$$
\begin{aligned}
|\Phi(u)| & \leq \gamma\|u\|_{1, p} \\
& \leq \hat{\gamma}\left(\left\|u_{0}\right\|_{1, p}+|u|_{1, p}\right) \\
& \leq \gamma_{1}+\frac{\beta}{2}|u|_{1, p}^{p} \\
& =\gamma_{1}+\frac{\beta}{2}\|F\|_{p}^{p}
\end{aligned}
$$

for some constant $\gamma_{1}>0$ which depends only on $\Omega, p, u_{0}$ and $\beta$. Thus (2.5) follows.

Lemma 2.2 : Let $W(x, F)$ satisfy (H1) - (H3). Let $\Phi(\cdot)$ be continuous in $W^{1, p}\left(\Omega ; R^{3}\right)$. Assume that

$$
\inf _{v \in A} I(v)<+\infty .
$$

Let $\left\{u_{j}\right\}$ be a minimizing sequence of $I(\cdot)$ in $A$.

Then, there exist a subsequence $\left\{u_{\nu}\right\}$ of $\left\{u_{j}\right\}$, functions $u \in A, \hat{E} \in$ $L^{s}\left(\Omega ; M^{3}\right)$ such that

$$
\begin{array}{rll}
u_{\nu} \rightarrow & u & \text { in } W^{1, p}\left(\Omega ; R^{3}\right), \\
F_{\nu} \rightarrow & F & \text { in } L^{p}\left(\Omega ; M^{3}\right), \\
\operatorname{adj} F_{\nu} \rightarrow & \text { adj } F & \text { in } L^{q}\left(\Omega ; M^{3}\right), \\
\operatorname{det} F_{\nu} \rightarrow \operatorname{det} F & \text { in } L^{r}(\Omega), \\
E_{\nu} \rightarrow \quad \hat{E} & \text { in } L^{s}\left(\Omega ; M^{3}\right),
\end{array}
$$

where $F_{\nu}=I+\nabla u_{\nu}, F=I+\nabla u$ and $E_{\nu}=\frac{1}{2}\left(F_{\nu}^{T} F_{\nu}-I\right)$.

Furthermore,

$$
\begin{aligned}
I(\hat{E}, u) & =\int_{\Omega} Z(x, \hat{E}, I+\nabla u) d x-\int_{\Omega} f \cdot u d x-\int_{\partial \Omega_{1}} g \cdot u d s, \\
& \leq \varliminf_{\nu \rightarrow \infty} I\left(u_{\nu}\right)=\inf _{v \in A} I(v) .
\end{aligned}
$$


Proof: It follows from lemma 2.1 and (2.8) that $\left\|E_{j}\right\|_{s},\left\|F_{j}\right\|_{p}$, $\left\|\operatorname{adj} F_{j}\right\|_{q}$ and $\left\|\operatorname{det} F_{j}\right\|_{r}, j=1,2, \cdots$ are bounded. Thus, there exist a subsequence $\left\{u_{\nu}\right\}$ of $\left\{u_{j}\right\}$, a function $u \in W^{1, p}\left(\Omega ; R^{3}\right)$ with $u=u_{0}$ on $\partial \Omega_{0}$, functions $H \in L^{q}\left(\Omega ; M^{3}\right), \delta \in L^{r}(\Omega)$ and $\hat{E} \in L^{s}\left(\Omega ; M^{3}\right)$ such that

$$
\begin{array}{rll}
u_{\nu} \rightarrow u & \text { in } & W^{1, p}\left(\Omega ; R^{3}\right), \\
F_{\nu} \rightarrow F & \text { in } & L^{p}\left(\Omega ; M^{3}\right), \\
\operatorname{adj} F_{\nu} \rightarrow H & \text { in } & L^{q}\left(\Omega ; M^{3}\right), \\
\operatorname{det} F_{\nu} \rightarrow \delta & \text { in } & L^{r}(\Omega) \\
E_{\nu} \rightarrow \hat{E} & \text { in } & L^{s}\left(\Omega ; M^{3}\right) .
\end{array}
$$

By the sequential weak continuity of Jacobians (see [6] [7]), we know that

$$
H=\operatorname{adj} F, \quad \delta=\operatorname{det} F .
$$

On the other hand, it follows from a standard lower semicontinuity theorem [16] that

$$
I(E, u)=\int_{\Omega} Z(x, E, I+\nabla u) d x-\int_{\Omega} f \cdot u d x-\int_{\partial \Omega_{1}} g \cdot u d s
$$

is sequentially weakly lower semicontinuous. Hence (2.14) follows. This and (H3) imply that

$$
\operatorname{det} F>0, \text { a.e. in } \Omega \text {. }
$$

Thus, $u \in A$. This completes the proof.

Remark 2.1 : Since $\hat{E}$ may not be equal to $\frac{1}{2}\left(F^{T} F-I\right)$, u obtained in lemma 2.2 may not be a minimizer of $I(\cdot)$ in $A$.

The following two lemmas [8] play an important role in the proof of theorem 2.1 .

Lemma 2.3 : Let $K \subset R^{M}$ be open and convex, and let $\Psi: K \rightarrow R$ be strictly convex. Let $0<\theta<1$, and suppose that $a_{j}, a \in K$ with

$\theta \Psi\left(a_{j}\right)+(1-\theta) \Psi(a)-\Psi\left(\theta a_{j}+(1-\theta) a\right) \rightarrow 0, \quad$ as $\quad j \rightarrow \infty$.

Then $a_{j} \rightarrow a$. 
Proof: We first note that

$$
h(b)=\theta \Psi(b)+(1-\theta) \Psi(a)-\Psi(\theta b+(1-\theta) a)
$$

is strictly increasing along any ray starting from $a$. In fact, let $e \in$ $R^{M}, 0<t_{1}<t_{2}$ such that $a+\xi t_{2} e \in K, \forall \xi \in[0,1]$, let $\alpha=\frac{t_{1}-\theta t_{1}}{t_{2}-\theta t_{1}}$, $\beta=\frac{\theta\left(t_{2}-t_{1}\right)}{t_{2}-\theta t_{1}}=(1-\alpha) \theta$, by

$$
\begin{aligned}
a+t_{1} e & =\alpha\left(a+t_{2} e\right)+(1-\alpha)\left(a+\theta t_{1} e\right) \\
a+\theta t_{2} e & =\beta\left(a+t_{2} e\right)+(1-\beta)\left(a+\theta t_{1} e\right)
\end{aligned}
$$

and the strict convexity of $\Psi$, we have

$$
\theta \Psi\left(a+t_{1} e\right)+\Psi\left(a+\theta t_{2} e\right)<\theta \Psi\left(a+t_{2} e\right)+\Psi\left(a+\theta t_{1} e\right) .
$$

This is equivalent to saying that

$$
h\left(a+t_{2} e\right)>h\left(a+t_{1} e\right), \quad 0<t_{1}<t_{2} .
$$

Since $K$ is open and $\Psi$ is convex, $\Psi$ is continuous. Therefore, given $\epsilon>0$ sufficiently small such that $\{|b-a| \leq \epsilon\} \subset K$, we have

$$
\inf _{|b-a|=\epsilon} h(b)>0 .
$$

Since $h$ is increasing along rays, this implies that

$$
\inf _{b \in K,|b-a| \geq \epsilon} h(b)>0,
$$

which gives the result.

Lemma 2.4 : Let $W_{j}, W, h_{j}, h, H_{j}, H \in L^{1}(\Omega)$ with $h_{j} \leq W_{j} \leq H_{j}$ for all $j$. Suppose that $W_{j} \rightarrow W, h_{j} \rightarrow h, H_{j} \rightarrow H$, a.e. in $\Omega$, and $\int_{\Omega} h_{j}(x) d x \rightarrow \int_{\Omega} h(x) d x, \int_{\Omega} H_{j}(x) d x \rightarrow \int_{\Omega} H(x) d x$ as $j \rightarrow \infty$.

Then

$$
\int_{\Omega} W_{j}(x) d x \rightarrow \int_{\Omega} W(x) d x .
$$

Proof: The result follows by applying Fatou's lemma to the sequences $H_{j}-W_{j}$ and $W_{j}-h_{j}$.

Theorem 2.1 : Let $W(x, F)$ satisfy hypothese (H1) - (H3). Let $\Phi(u)$ be continuous in $W^{1, p}\left(\Omega ; R^{3}\right)$. Let $\left\{u_{\nu}\right\}$ be a minimizing sequence of $I(\cdot)$ in $A$, and let $u \in A, \hat{E} \in L^{s}\left(\Omega ; M^{3}\right)$ be such that (2.9)-(2.14) hold.

Then, the statements (i) - (v) below are equivalent. 
(i) $\hat{E}=\frac{1}{2}\left(F^{T} F-I\right)$;

(ii) $I(\hat{E}, u)=\lim _{\nu \rightarrow \infty} I\left(u_{\nu}\right)=\inf _{v \in A} I(v)$;

(iii) $E_{\nu} \rightarrow \hat{E}$ in $L^{s}\left(\Omega ; M^{3}\right), F_{\nu} \rightarrow F$ in $L^{p}\left(\Omega ; M^{3}\right)$, adj $F_{\nu} \rightarrow \operatorname{adj} F$ in $L^{q}\left(\Omega ; M^{3}\right)$, and $\operatorname{det} F_{\nu} \rightarrow \operatorname{det} F$ in $L^{r}(\Omega)$;

(iv) $F_{\nu} \rightarrow F$ in $L^{p}\left(\Omega ; M^{3}\right)$;

(v) $F_{\nu} \rightarrow F$ in measure in $\Omega$.

Furthermore, $u \in A$ is a minimizer of $I(\cdot)$ in $A$ if any of (i) - (v) holds.

Proof: (a). Suppose (i) is true. Since $u \in A$, we have

$$
I(u)=I(\hat{E}, u) \geq \inf _{v \in A} I(v) .
$$

This and (2.14) give (ii).

(b). Suppose (ii) holds. Let

$$
\begin{aligned}
a(x) & =(\hat{E}(x), F(x), \operatorname{adj} F(x), \operatorname{det} F(x)), \\
a_{\nu}(x) & =\left(E_{\nu}(x), F_{\nu}(x), \operatorname{adj} F_{\nu}(x), \operatorname{det} F_{\nu}(x)\right) .
\end{aligned}
$$

For fixed $\theta \in(0,1)$, define

$$
h_{\nu}(x)=\theta G\left(x, a_{\nu}(x)\right)+(1-\theta) G(x, a(x))-G\left(x, \theta a_{\nu}(x)+(1-\theta) a(x)\right)
$$

It follows from (H1) that

$$
h_{\nu}(x) \geq 0, \quad \forall x \in \Omega .
$$

By lemma 2.2, we have

$$
\int_{\Omega} G(x, a(x)) d x \leq \underline{\lim }_{\nu \rightarrow \infty} \int_{\Omega} G\left(x, \theta a_{\nu}(x)+(1-\theta) a(x)\right) d x .
$$

Thus, by (ii)

$$
\begin{aligned}
0 & \leq \varlimsup_{\nu \rightarrow \infty} \int_{\Omega} h_{\nu}(x) d x \\
& =\int_{\Omega} G(x, a(x)) d x-\underline{\lim }_{\nu \rightarrow \infty} \int_{\Omega} G\left(x, \theta a_{\nu}(x)+(1-\theta) a(x)\right) d x \\
& \leq 0 .
\end{aligned}
$$


Thus, for a subsequence, again denoted by $a_{\nu}(x)$, we have

$$
h_{\nu} \rightarrow 0, \text { a.e. in } \Omega \text {. }
$$

Since $G(x, \cdot)$ is strictly convex (hypothesis (H1)), by lemma 2.3, we have

$$
a_{\nu}(x) \longrightarrow a(x), \quad \text { a.e. in } \Omega \text {. }
$$

Since $G(\cdot, \cdot)$ is continuous for almost all $x \in \bar{\Omega}$ (hypothesis (H1)), we also have

$$
G\left(x, a_{\nu}(x)\right) \rightarrow G(x, a(x)), \quad \text { a.e. in } \Omega .
$$

By (H2),

$$
\begin{aligned}
0 \leq & \left|\hat{E}(x)-E_{\nu}(x)\right|^{s}+\left|F(x)-F_{\nu}(x)\right|^{p}+ \\
& \left|a d j F(x)-\operatorname{adj} F_{\nu}(x)\right|^{q}+\left|\operatorname{det} F(x)-\operatorname{det} F_{\nu}(x)\right|^{r} \\
\leq & \hat{C}_{1}\left(G\left(x, a_{\nu}(x)\right)+G(x, a(x))\right)+\hat{C}_{2}
\end{aligned}
$$

for some constants $\hat{C}_{1}>0$ and $\hat{C}_{2} \in R$. Thus, by lemma 2.4 , we have

$$
\left\|\hat{E}-E_{\nu}\right\|_{s}^{s}+\left\|F-F_{\nu}\right\|_{p}^{p}+\left\|\operatorname{adj} F-\operatorname{adj} F_{\nu}\right\|_{q}^{q}+\left\|\operatorname{det} F-\operatorname{det} F_{\nu}\right\|_{r}^{r} \rightarrow 0 .
$$

Hence (iii), (iv) and (v) must be true.

(c). Suppose (iv) holds. Then,

$$
E_{\nu}=\frac{1}{2}\left(F_{\nu}^{T} F_{\nu}-I\right) \rightarrow \frac{1}{2}\left(F^{T} F-I\right), \quad \text { in } L^{s}\left(\Omega ; M^{3}\right) .
$$

Since $E_{\nu} \rightarrow \hat{E}$ in $L^{s}\left(\Omega ; M^{3}\right)$ by $(2.13)$, we conclude that $\hat{E}=\frac{1}{2}\left(F^{T} F-I\right)$, i.e. (i) holds.

(d). Suppose now (v) is true. Then, by Riesz's theorem for a subsequence, again denoted by $\left\{F_{\nu}\right\}$, we have

$$
\begin{array}{ll}
F_{\nu} \rightarrow F, & \text { a.e. in } \Omega, \\
E_{\nu} \rightarrow \frac{1}{2}\left(F^{T} F-I\right), & \text { a.e. in } \Omega .
\end{array}
$$

Thus, by (2.13), we have $\hat{E}=\frac{1}{2}\left(F^{T} F-I\right)$, i.e. (i) holds.

The arguments in (a) - (d) show that (i) - (v) are equivalent. Thus, if any of (i) - (v) is known to be true, then (i) - (v) all hold. Now it follows from $u \in A$ and (i) that

$$
I(u)=I(\hat{E}, u) \geq \inf _{v \in A} I(v) .
$$

This and (2.14) show that $u \in A$ is a minimizer of $I(\cdot)$ in $A$. 
Theorem 2.2 : Let $W(x, F)$ satisfy hypotheses (H1) - (H3). Let $\Phi(u)$ be continuous in $W^{1, p}\left(\Omega ; R^{3}\right)$. Let $\left\{u_{j}\right\}$ be a minimizing sequence of $I(\cdot)$ in A such that (2.9)-(2.14) hold for some $(u, \hat{E}) \in A \times L^{s}\left(\Omega ; M^{3}\right)$. Suppose

$$
\hat{E} \neq \frac{1}{2}\left((I+\nabla u)^{T}(I+\nabla u)-I\right) .
$$

Then, $\left\{u_{j}\right\}$ gives microstructure, i.e. there exists a measurable subset $\hat{\Omega} \subset \Omega$ with meas $(\hat{\Omega})>0$ such that

$$
\underline{\lim }_{j \rightarrow \infty} \int_{\Omega^{\prime}}\left|\nabla u_{j}-\nabla u\right| d x>0,
$$

and

$$
\lim _{j \rightarrow \infty} \int_{\Omega^{\prime}}\left(\nabla u_{j}-\nabla u\right) d x=0,
$$

for any measurable subset $\Omega^{\prime} \subset \hat{\Omega}$ with meas $\left(\Omega^{\prime}\right)>0$.

Proof: Let

$$
\hat{\Omega}=\left\{x \in \Omega: \hat{E}(x) \neq \frac{1}{2}\left((I+\nabla u(x))^{T}(I+\nabla u(x))-I\right)\right\} .
$$

By $(2.15)$, meas $(\hat{\Omega})>0$.

(2.17) is obvious, since $u_{j} \rightarrow u$ in $W^{1, p}\left(\Omega ; R^{3}\right)$.

Suppose that the theorem is not true. Then, there would exist a measurable subset $\Omega^{\prime} \subset \hat{\Omega}$ with meas $\left(\Omega^{\prime}\right)>0$ and a subsequence of $\left\{u_{j}\right\}$, again denoted by $\left\{u_{j}\right\}$, such that

$$
\lim _{j \rightarrow \infty} \int_{\Omega^{\prime}}\left|\nabla u_{j}-\nabla u\right| d x=0 .
$$

By extracting a further subsequence, we would then be able to find a subsequence $\left\{u_{\nu}\right\}$ of $\left\{u_{j}\right\}$ satisfying

$$
\nabla u_{\nu} \rightarrow \nabla u, \text { a.e. in } \Omega^{\prime},
$$

and thus

$$
\begin{gathered}
E_{\nu}=\frac{1}{2}\left(\left(I+\nabla u_{\nu}\right)^{T}\left(I+\nabla u_{\nu}\right)-I\right) \rightarrow \quad \frac{1}{2}\left((I+\nabla u)^{T}(I+\nabla u)-I\right) \\
\text { a.e. in } \Omega^{\prime} .
\end{gathered}
$$

This would imply

$$
\hat{E}=\frac{1}{2}\left((I+\nabla u)^{T}(I+\nabla u)-I\right), \text { a.e. in } \Omega^{\prime},
$$


since $E_{\nu} \rightarrow \hat{E}$ in $L^{s}\left(\Omega ; M^{3}\right)$ and hence also in $L^{s}\left(\Omega^{\prime} ; M^{3}\right)$. But this contradicts the assumption that $\Omega^{\prime} \subset \hat{\Omega}$.

As a corollary of theorem 2.1 and theorem 2.2, we have the following existence theorem for the problem of minimizing $I(\cdot)$ in $A$.

Theorem 2.3 : Let $W(x, F)$ satisfy hypotheses (H1) - (H3). Let $\Phi(u)$ be continuous in $W^{1, p}\left(\Omega ; R^{3}\right)$. Let $I(\cdot)$ and $A$ be defined by (2.2) and (2.3) respectively, and $\inf _{v \in A} I(v)<+\infty$.

Then, either there exists a minimizer of $I(\cdot)$ in $A$ or there exists a minimizing sequence of $I(\cdot)$ in A which leads to microstructure.

Proof: By lemma 2.2, there always exists a minimizing sequence $\left\{u_{\nu}\right\}$ of $I(\cdot)$ in $A$ such that $(2.9)$ - (2.14) hold for some functions $(u, \hat{E}) \in$ $A \times L^{s}\left(\Omega ; M^{3}\right)$. We have either

$$
\hat{E}=\frac{1}{2}\left((I+\nabla u)^{T}(I+\nabla u)-I\right),
$$

or

$$
\hat{E} \neq \frac{1}{2}\left((I+\nabla u)^{T}(I+\nabla u)-I\right)
$$

In the former case, by theorem $2.1, u \in A$ is a minimizer of $I(\cdot)$ in $A$. While in the later case, by theorem $2.2,\left\{u_{\nu}\right\}$ leads to microstructure.

Theorem 2.4 : Let $W(x, F)$ satisfy hypotheses (H1) - (H3). Let $\Phi(u)$ be continuous in $W^{1, p}\left(\Omega ; R^{3}\right)$. Let $\left\{u_{\nu}\right\}$ be a minimizing sequence of $I(\cdot)$ in $A$ such that (2.9) - (2.14) hold for some functions $(u, \hat{E}) \in A \times$ $L^{s}\left(\Omega ; M^{3}\right)$.

Then, a necessary and sufficient condition for $u$ to be a minimizer of $I(\cdot)$ in $A$ and $\hat{E}=\frac{1}{2}\left((I+\nabla u)^{T}(I+\nabla u)-I\right)$ is that

$$
\nabla u_{\nu} \rightarrow \nabla u, \quad \text { in measure in } \Omega
$$

and a necessary and sufficient condition for $\left\{u_{\nu}\right\}$ to lead to microstructure is that

$$
\underline{\lim }_{\nu \rightarrow \infty}\left\|u_{\nu}-u\right\|_{1, p, \Omega}>0
$$

Proof: The first conclusion follows directly from theorem 2.1. The second conclusion follows from theorem 2.2 and the definition of the microstructure. 
Remark 2.2 : In the case when $G(x, \cdot, \cdot, \cdot)$ is convex but not strictly convex, the existence of a solution in the form of a minimizer or microstructure can still be established. Similarly, we can prove that a minimizing sequence $\left\{u_{j}\right\}$ leads to microstructure if $\hat{E} \neq \frac{1}{2}\left((I+\nabla u)^{T}(I+\right.$ $\nabla u)-I$ ) and converges to a minimizer if $\hat{E}=\frac{1}{2}\left((I+\nabla u)^{T}(I+\nabla u)-I\right)$.

Remark 2.3 : The theory can also be easily extended to the stored energy function of the form $W(x, E, F)=G(x, E, \operatorname{adj} E$, det $E, F, \operatorname{adj} F$, $\operatorname{det} F)$ with $G(x, \cdot, \cdot, \cdot, \cdot, \cdot, \cdot)$ being continuous, convex and satisfying certain growth and coerceiveness conditions similar to (H2) and (H3).

Remark 2.4 It follows from the same arguments that all the theorems in this section remain valid if $W(x, F)$ is everywhere finite in $\Omega \times M^{3}$, and the hypothesis (H3) and the restriction on the admissible functions A that $\operatorname{det} F>0$, a.e. in $\Omega$ are removed and hypotheses (H1) and (H2) are replaced by the following hypotheses

$\left(\mathbf{H 1}^{\prime}\right)$ There is a Carathéodory function $G: \bar{\Omega} \times M^{3} \times M^{3} \times M^{3} \times$ $R \rightarrow R$, i.e. $G(x, \cdot, \cdot, \cdot, \cdot)$ is continuous for almost all $x \in \bar{\Omega}$ and $G(\cdot, E, F, H, \delta)$ is measurable for all $(E, F, H, \delta) \in M^{3} \times M^{3} \times$ $M^{3} \times R$, such that $G(x, \cdot, \cdot, \cdot, \cdot)$ is strictly convex with respect to explicitly dependent variables and

$W(x, F)=G(x, E, F, \operatorname{adj} F, \operatorname{det} F), \quad$ for all $x \in \bar{\Omega}$ and $F \in M^{3}$.

$\left(\mathbf{H 2}^{\prime}\right)$ There are constants $C_{0} \in R, C_{1}>0, s>1, p \geq 2 s, q \geq \frac{p}{p-1}$, $r>1$ such that

$$
G(x, E, F, H, \delta) \geq C_{0}+C_{1}\left(|E|^{s}+|F|^{p}+|H|^{q}+|\delta|^{r}\right),
$$

for $\operatorname{all}(x, E, F, H, \delta) \in \bar{\Omega} \times M^{3} \times M^{3} \times M^{3} \times R$.

This allows us to cover St. Venant Kirchhoff materials which are important in applications. In fact, we only need to take $s=2, p=4, q=$ 2 , and $r=4 / 3$ in hypothesis (H2') and apply the theorems in the adjusted form (see also §3). 


\section{Application to nonlinear elasticity}

Let $\Omega \subset R^{3}$ be a natural state of an elastic material which is isotropic and material frame indifferent. Let $W(F)$ be the stored energy function of the material. Then, by a standard result in elasticity [3], if $W(\cdot) \in$ $C^{1}\left(M_{+}^{3} ; R\right)$,

$$
W(F) \equiv W^{*}(E)=\frac{\lambda}{2}(\operatorname{tr} E)^{2}+\mu t r E^{2}+o\left(|E|^{2}\right),
$$

where $\lambda>0, \mu>0$ are constants.

When the displacement is small, i.e. $|\nabla u| \ll 1$, which is the case in pure displacement boundary value problems when $\partial \Omega$ is sufficiently smooth and the body force $f$ is sufficiently small [3], the stored energy function of St. Venant-Kirchhoff materials

$$
W_{1}(E)=\frac{\lambda}{2}(\operatorname{tr} E)^{2}+\mu \operatorname{tr} E^{2}
$$

is a good approximation of the stored energy function in the form of (3.1). However, for general boundary value problems, $\nabla u$ is in general not small, and the effect of $o\left(|E|^{2}\right)$ must then be taken into account.

A natural condition that a stored energy function must satisfy is that [6] [7]

$$
W(F) \rightarrow+\infty, \quad \text { as } \quad \operatorname{det} F \rightarrow 0^{+} .
$$

Now, we consider the stored energy function of the form

$$
W(F)=W_{1}(E)+W_{2}(F),
$$

where $W_{1}(E)$ is defined by $(3.2), W_{2}(F)$ satisfies (H1) in $\S 2$ and

$$
\begin{aligned}
& W_{2}(F) \geq C, \quad \text { for some constant } \quad C \in R, \\
& W_{2}(F)=o\left(|E|^{2}\right), \\
& W_{2}(F) \rightarrow+\infty \quad \text { as } \quad \operatorname{det} F \rightarrow 0^{+} .
\end{aligned}
$$

Lemma 3.1 : Let $W(F)$ be of the form (3.3) with $W_{1}(E)$ being defined by (3.2) and $W_{2}(F)$ satisfying (H1) in $\S 2$ and (3.4) - (3.6). Then $W(F)$ satisfies (H1)-(H3) for $s=2, p=4, q=2$ and $r=\frac{4}{3}$.

Proof: Since $W_{2}(F)$ satisfies (H1), there is a continuous function $G_{2}: M^{3} \times M^{3} \times M^{3} \times R_{+} \rightarrow R$ such that $G_{2}(\cdot, \cdot, \cdot, \cdot)$ is strictly convex,

$$
W_{2}(F)=G_{2}(E, F, \operatorname{adj} F, \operatorname{det} F), \quad \forall F \in M_{+}^{3},
$$


where $E=\frac{1}{2}\left(F^{T} F-I\right)$, and (3.6) holds.

Define $G: M^{3} \times M^{3} \times M^{3} \times R_{+} \rightarrow R$ by

$$
G(E, F, H, \delta)=W_{1}(E)+G_{2}(E, F, H, \delta)
$$

Then, it is obvious that $W(F)$ satisfies (H1) and (H3) with $G(\cdot, \cdot, \cdot, \cdot)$ defined by (3.8). It follows from

$$
\begin{aligned}
|E|^{2} & =\operatorname{tr} E^{2}, & \\
|F|^{4} & =(3+2 \operatorname{tr} E)^{2} \leq & 45+5(\operatorname{tr} E)^{2}, \\
|\operatorname{adj} F|^{2} & \leq \hat{C}|F|^{4}, & \text { for some constant } \hat{C}>0, \\
|\operatorname{det} F|^{\frac{4}{3}} & \leq \hat{C}|F|^{4}, & \text { for some constant } \hat{C}>0,
\end{aligned}
$$

that (H2) holds.

Theorem 3.1 : Let $W(F)$ satisfy the hypotheses in lemma 3.1. Let $\Phi(\cdot)$ defined by (2.4) be continuous in $W^{1,4}\left(\Omega ; R^{3}\right)$. Let $I(\cdot)$ be defined by (2.2) and

$$
\begin{gathered}
A=\left\{u \in W^{1,4}\left(\Omega ; R^{3}\right): F=I+\nabla u, \operatorname{det} F>0, \text { a.e. in } \Omega,\right. \\
\text { and } \left.u=u_{0}, \text { on } \partial \Omega_{0}\right\} .
\end{gathered}
$$

Suppose

$$
\inf _{v \in A} I(v)<+\infty
$$

Then, for any minimizing sequence $\left\{u_{j}\right\}$ of $I(\cdot)$ in $A$, there exist a subsequence $\left\{u_{\nu}\right\}$ of $\left\{u_{j}\right\}$, a function $u \in A$ and a function $\hat{E} \in$ $L^{2}\left(\Omega ; M^{3}\right)$ satisfying

$$
\begin{array}{lll}
u_{\nu} \rightarrow u & \text { in } & W^{1,4}\left(\Omega ; R^{3}\right), \\
E_{\nu} \rightarrow \hat{E} & \text { in } & L^{2}\left(\Omega ; M^{3}\right),
\end{array}
$$

such that either

$$
\begin{array}{rc}
u_{\nu} \rightarrow u & \text { in } \quad W^{1,4}\left(\Omega ; R^{3}\right), \\
E_{\nu} \rightarrow E & =\frac{1}{2}\left((I+\nabla u)^{T}(I+\nabla u)-I\right) \quad \text { in } \quad L^{2}\left(\Omega ; M^{3}\right),
\end{array}
$$

or $\left\{u_{\nu}\right\}$ oscillates more and more finely and leads to microstructure. 
Proof: The existence of $\left\{u_{\nu}\right\}, u$ and $\hat{E}$ satisfying (3.11) and (3.12) follows from lemma 2.2. The rest of the theorem is a corollary of theorem 2.1 and theorem 2.2 .

As an example, we may take

$$
W_{2}(F)=\alpha\left|(\operatorname{det} F)^{2}-1\right|^{r_{1}}|\log (\operatorname{det} F)|,
$$

where $\alpha>0, r_{1}>1$ are constants. Notice that

$$
(\operatorname{det} F)^{2}-1=2(\operatorname{tr} E+2 \operatorname{tr}(\operatorname{adj} E)+4 \operatorname{det} E),
$$

we have

$$
\left|W_{2}(F)\right| \leq C|E|^{r_{1}-1} \frac{|\log (1+C|E|)|}{|E|}|E|^{2}=o\left(|E|^{2}\right) .
$$

It is obvious that $W_{2}(F) \geq 0$. Let $G_{2}(\delta)=\alpha\left|\delta^{2}-1\right|^{r_{1}}|\log \delta|$, then it is easy to show that $W_{2}(F)$ satisfies (H1) and (3.6). Thus for

$$
W(F)=\frac{\lambda}{2}(\operatorname{tr} E)^{2}+\mu \operatorname{tr} E^{2}+\alpha\left|(\operatorname{det} F)^{2}-1\right|^{r_{1}}|\log (\operatorname{det} F)|,
$$

theorem 3.1 holds.

\section{References}

[1] J.M. Ball and R.D. James, Fine phase mixtures as minimizers of energy, Arch. Rat. Mech. Anal., 100(1987), 13-52.

[2] P.J. Swart and P.J. Holmes, Energy minimization and the formation of microstructure in dynamic anti-plane shear, Arch. Rat. Mech. Anal., 121(1992), 37-85.

[3] P.G. Ciarlet, Mathematical Elasticity, North-Holland, Amsterdam, 1988.

[4] Z.-P. Li, The finite element method for nonlinear elasticity, J. Comput. Math., 7(1)(1989), 1-14.

[5] Z.-P. Li, The finite element incremental method for nonlinear elasticity, Northeastern Math. J., 6(1)(1990), 93-100.

[6] J.M. Ball, Convexity conditions and existence theorems in nonlinear elasticity. Arch. Rat. Mech. Anal., 63(1977), 337-403. 
[7] J.M. Ball, Constitutive inequalities and existence theorems in nonlinear elastostatics, in "Nonlinear Analysis and Mechanics: Heriot-Watt Symposium Vol.1" (edited by R.J. Knops), Pitman, London, 1977.

[8] J.M. Ball and J.E. Marsden, Quasiconvexity at the boundary , positivity of the second variation, and elastic stability, Arch. Rat. Mech. Anal., 86(1984), 251-277.

[9] A. Raoult, Non-polyconvexity of the stored energy functions of a St. Venant-Kirchhoff material, Aplikace Matematiky 31 (1986), 417-419.

[10] A.C. Pipkin, Relaxed energy densities for small deformations of membranes, IMA J. Appl. Math., 50(1993), 225-237.

[11] A.C. Pipkin, Relaxed energy densities for large deformations of membranes, to appear in IMA J. Appl. Math..

[12] H. Le Dret and A. Raoult, The nonlinear membrane model as variational limit of nonlinear three-dimensional elasticity, to appear in J. Math. Pure Appl..

[13] H. Le Dret and A. Raoult, Envelope quasi-convexe de la densité d'énergie de Saint Venant-Kirchhoff, C.R. Acad. Sci. Paris, t.318, Série I (1994), 93-98.

[14] H. Le Dret and A. Raoult, Remarks on the quasiconvex envelope of stored energy functions in nonlinear elasticity, to appear in Commu. Appl. Nonlinear Anal..

[15] R.A. Adams, Sobolev Spaces, Academic Press, New York, 1975.

[16] A.D. Ioffe, On lower semicontinuity of integral functionals, I and II, SIAM J. Control and Optimization, 15(1977), 521-538, 991-1000. 